\title{
Existence of solutions for perturbed fractional equations with two competing weighted nonlinear terms
}

\author{
Yujuan Tian ${ }^{1 *}$ and Shasha Zhao'
}

\section{"Correspondence:}

tianyujuan0302@126.com 'School of Mathematics and Statistics, Shandong Normal University, Jinan, China

\section{Springer}

\begin{abstract}
This paper deals with a perturbed nonlocal equation of fractional Laplacian type involving two competing nonlinear terms with weights $f$ and $h$. Under two kinds of integrability conditions on the ratio of $f$ to $h$, we show some different existence results in this setting by using variational methods. Our results are extension of some problems studied by Carboni and Mugnai (J. Differ. Equ. 262(3):2393-2413, 2017) and Xiang et al. (J. Differ. Equ. 260(2):1392-1413, 2016).

MSC: 35A01;35J20; 35J60; 35R11

Keywords: Fractional Laplacian; Weighted nonlinearities; Variational methods; Existence
\end{abstract}

\section{Introduction and main results}

The aim of this paper is to derive the existence and multiplicity of nontrivial solutions for the following one-parameter perturbed nonlocal problem:

$$
\begin{cases}(-\Delta)^{s} u+v(x) u=\lambda f(x)|u|^{q-2} u-h(x) g(x, u) & \text { in } \Omega, \\ u=0 & \text { in } \mathbb{R}^{N} \backslash \Omega,\end{cases}
$$

where $\lambda \in \mathbb{R}, 0<s<1, \Omega \subset \mathbb{R}^{N}$ is a bounded domain with Lipschitz boundary, $N>2 s$. Here $(-\Delta)^{s}$ is the fractional Laplacian operator defined, up to a normalization factor, by

$$
(-\Delta)^{s} u(x)=-\frac{1}{2} \int_{\mathbb{R}^{N}} \frac{u(x+y)+u(x-y)-2 u(x)}{|y|^{N+2 s}} d y, \quad x \in \mathbb{R}^{N}
$$

along any rapidly decaying function $u$ of class $C^{\infty}\left(\mathbb{R}^{N}\right)$; see [2]. Such operators arise in a quite natural way in many different contexts, such as the thin obstacle problem, optimization, finance, probability, and materials science (see [3-6]).

In recent years, great attention has been focused on the study of the problems involving fractional operators, both for pure mathematical research and for concrete applications. Here we only collect some results for problems with two competing nonlinear terms. Local problems with combined effect of convex and concave nonlinearities have been widely

(c) The Author(s) 2018. This article is distributed under the terms of the Creative Commons Attribution 4.0 International License (http://creativecommons.org/licenses/by/4.0/), which permits unrestricted use, distribution, and reproduction in any medium, provided you give appropriate credit to the original author(s) and the source, provide a link to the Creative Commons license, and indicate if changes were made. 
investigated in the past decades; see, for example, [7-12]. In particular, Alama and Tarantello [10] considered the convex-concave Dirichlet problem

$$
\begin{cases}-\Delta u=\lambda u+f(x) u^{q-1}-h(x) u^{r-1} & \text { in } \Omega \\ u>0 & \text { in } \Omega, \\ u=0 & \text { on } \partial \Omega\end{cases}
$$

where $2<q<r$, and $f$ and $h$ are nonnegative weights. They first proved that the existence and multiplicity of solutions depend both on $\lambda$ and the integrability of a certain ratio of $f$ to $h$. Related problems with a perturbed term in the whole space were treated by Autuori and Pucci [12], who studied the following quasilinear equation:

$$
-\operatorname{div}\left(|\nabla u|^{p-2} \nabla u\right)+a(x)|u|^{p-2} u=\lambda f(x)|u|^{q-2} u-h(x)|u|^{r-2} u \quad \text { in } \mathbb{R}^{N},
$$

where $\max \{2, p\}<q<\min \left\{r, \frac{p N}{N-p}\right\}$, and the weights $f$ and $h$ are related by the integrability condition $\left(\frac{f^{r}}{h^{q}}\right)^{\frac{1}{r-q}} \in L^{1}\left(\mathbb{R}^{N}\right)$. After that, in [13], they extended these results to a nonlocal problem involving the fractional Laplacian and indicated that it is still an open problem when the integrability condition on $f$ and $h$ is replaced by the weaker assumption $\left(\frac{f^{r-2}}{h^{q-2}}\right)^{\frac{1}{r-q}} \in L^{\frac{N}{2 s}}\left(\mathbb{R}^{N}\right)$. Xiang et al. [1] gave a positive answer to this open problem in the setting of fractional $p$-Laplacian in $\mathbb{R}^{N}$. In these papers, to obtain the existence of solutions, the authors assume that perturbed weights $a(x)$ are bounded below by positive constants or positive functions.

All these problems involve convex-concave nonlinearities. Rădulescu and Repovš [11] dealt with the concave-concave Dirichlet problem without perturbed term in a bounded domain:

$$
\begin{cases}-\Delta u=\lambda f(x) u^{q-1}-h(x) u^{r-1} & \text { in } \Omega, \\ u>0 & \text { in } \Omega, \\ u=0 & \text { on } \partial \Omega .\end{cases}
$$

Assuming that $1<q<2<r<\frac{2 N}{N-2}$ and $f, h \in L^{\infty}$ are such that $\operatorname{essinf}_{x \in \Omega} f(x)>0$ and $\operatorname{ess}_{\inf } x \in \Omega(x)>0$, they proved the nonexistence and multiplicity of solutions with respect to the different range of $\lambda$. When $f(x) \equiv 1$ in $\Omega$, a nonlocal version of problem (1.1) involving the square root of Laplacian $\left(s=\frac{1}{2}\right)$ was studied in [14] with $1<q<r<2_{s}^{*}=\frac{2 N}{N-2 s}$, $h \in L^{\frac{q}{q-r}}(\Omega)$, and $h \in L^{\sigma}(\Omega), \sigma>\frac{2_{s}^{*}}{2_{s}^{*}-r}$.

The equation considered in problem $\left(P_{\lambda}\right)$ is in spirit of the previous papers, even if most of them deal with problem not directly comparable to ours. The nonlinear terms in $\left(P_{\lambda}\right)$ are related to the main elliptic part by requesting that:

$\left(\mathrm{H}_{1}\right) g$ is a Carathéodory function, and there exist two positive constants $b_{1}$ and $b_{2}$ such that

$$
b_{1}|t|^{r} \leq g(x, t) t \leq b_{2}|t|^{r} \quad \text { for a.e. } x \in \Omega \text { and all } t \in \mathbb{R},
$$

where $1<q<r<2_{s}^{*}$, and $2_{s}^{*}=\frac{2 N}{N-2 s}$ is the Sobolev fractional critical exponent; 
$\left(\mathrm{H}_{2}\right) f, h: \Omega \rightarrow \mathbb{R}_{+}$are positive weights satisfying $f \in L^{\frac{2_{s}^{*}}{2_{s}^{*}}}(\Omega)$ and $h \in L^{\frac{2_{s}^{*}}{2-r}}(\Omega)$. Moreover, $f(x)$ and $h(x)$ are related by the condition

$$
\left(\frac{f^{r}}{h^{q}}\right)^{\frac{1}{r-q}} \in L^{1}(\Omega)
$$

$\left(\mathrm{H}_{3}\right) v: \Omega \rightarrow \mathbb{R}$ is a perturbed weight such that $v(x) \geq 0$ for a.e. $x \in \Omega$.

We now state our main results. Our first result covers both convex-concave and concave-concave cases.

Theorem 1.1 Suppose that assumptions $\left(\mathrm{H}_{1}\right)-\left(\mathrm{H}_{3}\right)$ are satisfied. Then there exist three numbers $\lambda^{*} \geq \lambda_{*} \geq \lambda_{0} \geq 0$ such that problem $\left(P_{\lambda}\right)$ has

(i) only the trivial solution if $\lambda<\lambda_{0}$;

(ii) at least one nontrivial solution of definite sign if $\lambda>\lambda_{*}$;

(iii) at least two nontrivial solutions if $\lambda>\lambda^{*}$; one them is positive, and one is negative.

As $q>2$, we replace (1.3) by the assumption

$$
\left(\frac{f^{r-2}}{h^{q-2}}\right)^{\frac{1}{r-q}} \in L^{\frac{N}{2 s}}(\Omega)
$$

Note that thanks to $q<2_{s}^{*}$, by the Hölder's inequality we have

$$
\left\|\left(\frac{f^{r-2}}{h^{q-2}}\right)^{\frac{1}{r-q}}\right\|_{L^{\frac{N}{2 s}(\Omega)}} \leq\|f\|_{L^{\frac{2}{q}}}^{\frac{2_{s}^{*}}{2_{s}^{*}-q}(\Omega)}\left\|\left(\frac{f^{r}}{h^{q}}\right)^{\frac{1}{r-q}}\right\|_{L^{1}(\Omega)}^{1-\frac{2}{q}},
$$

which means that requirement (1.4) is weaker than (1.3) (see also [1, 13]). In this situation, we relabel $\left(\mathrm{H}_{2}\right)$ as $\left(\mathrm{H}_{2}^{\prime}\right)$. We state some improved results as follows.

Theorem 1.2 Let $2<q<r<2_{s}^{*}$ and suppose that (1.2), $\left(\mathrm{H}_{2}^{\prime}\right)$, and $\left(\mathrm{H}_{3}\right)$ hold. Then

(i) the results of Theorem 1.1 still hold, and the number $\lambda_{0}>0$;

(ii) if $\lambda=\lambda_{0}$, then problem $\left(P_{\lambda}\right)$ has at least one nontrivial solution; if $\lambda=\lambda_{*}$, then problem $\left(P_{\lambda}\right)$ has at least one nontrivial solution of definite sign; if $\lambda=\lambda^{*}$, then problem $\left(P_{\lambda}\right)$ has at least two nontrivial solutions; one of them is positive, and one is negative;

(iii) there exist a number $\tilde{\lambda} \geq \lambda^{*}$ such that problem $\left(P_{\lambda}\right)$ has at least four nontrivial solutions for every $\lambda \geq \tilde{\lambda}$; two of them are positive, and two are negative.

Our work are an extension of problems studied in [14], where $s=\frac{1}{2}, f(x) \equiv 1$, and $v(x) \equiv 0$. Even in this case, our assumptions on $h(x)$ are weaker than those in [14] since $h \in L^{\frac{2 *}{22_{s}^{*}-r}}(\Omega)$, which is allowed to satisfy condition (1.4) here. On the other hand, the problem studied in this paper covers both convex-concave and concave-concave cases, and owing to the different approach used here, the assumptions on perturbed weight can be relaxed to be nonnegative. From this point, our results extend some results of [1]. In addition, using variational methods, we present more complete results in the convex-concave case by describing the problem at all threshold values $\lambda_{0}, \lambda_{*}, \lambda^{*}$, and $\tilde{\lambda}$. 
Many other types of fractional and nonlocal problems are also actively studied in recent years. For example, we refer to [15-21] for fractional Kirchhof-type problems. Studies of fractional Schrödinger problems can be found in [22-24]. Fractional problems with special nonlinearities have been considered in [25-27]. Results on the Brézis-Nirenberg problem for a fractional operator were presented in [28, 29].

The remaining of this paper is organized as follows. In Sect. 2, we recall some preliminary results and main embedding results for fractional Sobolev spaces. In Sect. 3 and 4, we prove Theorems 1.1 and 1.2, respectively.

\section{Preliminaries}

In this section, we recall some necessary definitions and properties of the fractional Laplacian operator. For further details, we refer to $[2,30,31]$ and references therein.

We denote by $H^{s}\left(\mathbb{R}^{N}\right)$ the classical fractional Sobolev space with the Gagliardo norm

$$
\|u\|_{H^{s}\left(\mathbb{R}^{N}\right)}=\|u\|_{L^{2}\left(\mathbb{R}^{N}\right)}+\left(\iint_{\mathbb{R}^{2 N}} \frac{|u(x)-u(y)|^{2}}{|x-y|^{N+2 s}} d x d y\right)^{\frac{1}{2}} .
$$

For a detailed account on the properties of $H^{s}\left(\mathbb{R}^{N}\right)$, we refer to [2]. In this paper, we consider the closed linear subspace

$$
X_{0}^{s}(\Omega)=\left\{u \in H^{s}\left(\mathbb{R}^{N}\right): u(x)=0 \text { for a.e. } x \in \mathbb{R}^{N} \backslash \Omega\right\}
$$

with the norm

$$
\|u\|_{X_{0}^{s}(\Omega)}=\left(\iint_{\mathbb{R}^{2 N}} \frac{|u(x)-u(y)|^{2}}{|x-y|^{N+2 s}} d x d y\right)^{\frac{1}{2}},
$$

which is equivalent to its general norm (see [2], Theorem 7.1). Then $X_{0}^{s}(\Omega)$ is a Hilbert space. Let the space $E$ denote the completion of $C_{0}^{\infty}(\Omega)$ with respect to the norm

$$
\|u\|_{E}=\left(\|u\|_{X_{0}^{s}(\Omega)}^{2}+\int_{\Omega} v(x)|u(x)|^{2} d x\right)^{\frac{1}{2}} .
$$

It is readily seen that $E$ is a convex Banach space with scalar product

$$
\langle u, \varphi\rangle_{E}=\iint_{\mathbb{R}^{2 N}} \frac{(u(x)-u(y))(\varphi(x)-\varphi(y))}{|x-y|^{N+2 s}} d x d y+\int_{\Omega} v(x) u(x) \varphi(x) d x
$$

and has the following embedding property.

Lemma 2.1 Let $1 \leq \theta \leq 2_{s}^{*}$. Then we have the continuous embedding

$$
E \hookrightarrow L^{\theta}(\Omega)
$$

and there exists constants $C_{\theta}$ such that

$$
\|u\|_{\theta} \leq C_{\theta}\|u\|_{E} \quad \text { for all } u \in E
$$

In particular, for $\theta \in\left[1,2_{s}^{*}\right)$, the embedding is compact. 
Proof It is easy to see that the embedding $E \hookrightarrow X_{0}^{s}(\Omega)$ is continuous with $\|u\|_{X_{0}^{s}(\Omega)} \leq\|u\|_{E}$. By Theorem 7.1 in [2] the embedding $X_{0}^{s}(\Omega) \hookrightarrow L^{\theta}(\Omega)$ is continuous for all $1 \leq \theta \leq 2_{s}^{*}$ with

$$
\|u\|_{\theta} \leq C_{\theta}\|u\|_{X_{0}^{s}(\Omega)}
$$

where $C_{\theta}$ is a positive constant. Moreover, the embedding is compact when $\theta \in\left[1,2_{s}^{*}\right)$. So, we get the claim.

On the other hand, for any $r, q \geq 1$, we introduce the Banach spaces

$$
L_{f}^{q}(\Omega)=\left\{u \in L_{\mathrm{loc}}^{1}(\Omega): \int_{\Omega} f(x)|u|^{q} d x<\infty\right\}
$$

equipped with the norms

$$
\|u\|_{f, q}=\left(\int_{\Omega} f(x)|u|^{q} d x\right)^{\frac{1}{q}}
$$

and

$$
L_{h}^{r}(\Omega)=\left\{u \in L_{\mathrm{loc}}^{1}(\Omega): \int_{\Omega} h(x)|u|^{r} d x<\infty\right\}
$$

equipped with the norm

$$
\|u\|_{h, r}=\left(\int_{\Omega} h(x)|u|^{r} d x\right)^{\frac{1}{r}} .
$$

Lemma 2.2 Assume that $1 \leq r, q<2_{s}^{*}, f \in L^{\frac{2_{s}^{*}}{2 s}}(\Omega)$, and $h \in L^{\frac{2_{s}^{*}}{2 s_{s}^{*}-r}}(\Omega)$. Then we have the compact embeddings

$$
E \hookrightarrow L_{h}^{r}(\Omega)
$$

and

$$
E \hookrightarrow L_{f}^{q}(\Omega)
$$

and there exist constants $C_{h}$ and $C_{f}$ such that

$$
\|u\|_{h, r} \leq C_{h}\|u\|_{E}
$$

and

$$
\|u\|_{f, q} \leq C_{f}\|u\|_{E}
$$

The lemma can be proved similarly as Theorem 2.1 in [1], which we will not repeat here. Now, in view of the previous considerations, we have the definition of weak solution to problem $\left(P_{\lambda}\right)$. 
Definition 2.1 We say that $u \in E$ is a weak solution to problem $\left(P_{\lambda}\right)$ if

$$
\begin{aligned}
& \iint_{\mathbb{R}^{2 N}} \frac{(u(x)-u(y))(\varphi(x)-\varphi(y))}{|x-y|^{N+2 s}} d x d y+\int_{\Omega} v(x) u(x) \varphi(x) d x \\
& \quad=\lambda \int_{\Omega} f(x)|u|^{q-2} u \varphi d x-\int_{\Omega} h(x) g(x, u) \varphi d x
\end{aligned}
$$

for every $\varphi \in E$.

Finally, let us recall an inequality that we will use afterward.

Lemma 2.3 (see [1]) For any $k_{1}>0, k_{2}>0$, and $0<\eta<\beta$, we have

$$
k_{1}|t|^{\eta}-k_{2}|t|^{\beta} \leq k_{1}\left(\frac{k_{1}}{k_{2}}\right)^{\frac{\eta}{\beta-\eta}} \quad \text { for all } t \in \mathbb{R} .
$$

\section{Proof of Theorem 1.1}

The weak solutions of $\left(P_{\lambda}\right)$ correspond to the critical points of the energy functional $\Psi$ : $E \rightarrow \mathbb{R}$ given by

$$
\begin{aligned}
\Psi(u)= & \frac{1}{2} \iint_{\mathbb{R}^{2 N}} \frac{|u(x)-u(y)|^{2}}{|x-y|^{N+2 s}} d x d y+\frac{1}{2} \int_{\Omega} v(x) u^{2} d x \\
& -\frac{\lambda}{q} \int_{\Omega} f(x)|u|^{q} d x+\int_{\Omega} h(x) G(x, u) d x,
\end{aligned}
$$

where

$$
G(x, t)=\int_{0}^{t} g(x, \tau) d \tau
$$

By condition $\left(\mathrm{H}_{1}\right)$ we deduce

$$
\frac{b_{1}}{r}|t|^{r} \leq G(x, t) \leq \frac{b_{2}}{r}|t|^{r} \quad \text { for a.e. } x \in \Omega \text { and all } t \in \mathbb{R} .
$$

\subsection{Nonexistence of solutions}

Let $\lambda_{0}=\inf \left\{\lambda \in \mathbb{R}:\right.$ problem $\left(P_{\lambda}\right)$ admits at least a nontrivial solution $\}$. Then $\lambda_{0}$ exists (see Sect. 3.2), and $\lambda_{0} \geq 0$. Indeed, assuming that $u \in E$ is a nontrivial solution of problem $\left(P_{\lambda}\right)$ and taking the test function $\varphi=u$ in (2.1), we have

$$
0<\|u\|_{E}^{2}+\int_{\Omega} h(x) g(x, u) u d x=\lambda \int_{\Omega} f(x)|u|^{q} d x
$$

which implies $\lambda>0$. Thus $\lambda_{0} \geq 0$, and the proof of (i) is completed.

\subsection{Existence of solutions}

From now on, assume that $\lambda>0$ and denote $u^{+}=\max \{u, 0\}$ and $u^{-}=\max \{-u, 0\}$. We consider the following "positive" and "negative" truncations of the functional $\Psi$ :

$$
\Psi_{+}(u)=\frac{1}{2}\|u\|_{E}^{2}-\frac{\lambda}{q} \int_{\Omega} f(x)\left|u^{+}\right|^{q} d x+\int_{\Omega} h(x) G\left(x, u^{+}\right) d x
$$


and

$$
\Psi_{-}(u)=\frac{1}{2}\|u\|_{E}^{2}-\frac{\lambda}{q} \int_{\Omega} f(x)\left|u^{-}\right|^{q} d x+\int_{\Omega} h(x) G\left(x,-u^{-}\right) d x
$$

for $u \in E$.

Obviously, if $u$ is a critical point of functional $\Psi_{+}$, then for all $\varphi \in E$, we have

$$
\langle u, \varphi\rangle_{E}-\lambda \int_{\Omega} f(x)\left|u^{+}\right|^{q-2} u^{+} \varphi d x+\int_{\Omega} h(x) g\left(x, u^{+}\right) \varphi d x=0,
$$

whereas if $u$ is a critical point of $\Psi_{-}$, then for all $\varphi \in E$, we have

$$
\langle u, \varphi\rangle_{E}-\lambda \int_{\Omega} f(x)\left|u^{-}\right|^{q-2}\left(-u^{-}\right) \varphi d x+\int_{\Omega} h(x) g\left(x,-u^{-}\right) \varphi d x=0 .
$$

Step 1. We prove that there exist a nonnegative critical point $u_{1}$ for the functional $\Psi_{+}$ and a nonpositive critical point $u_{2}$ for the functional $\Psi_{-}$.

Let us first focus on the functional $\Psi_{+}$. We have the following lemma.

Lemma 3.1 The functional $\Psi_{+}$is coercive and weakly lower semicontinuous in $E$.

Proof For any $u \in E$, from (3.2) we have that

$$
\Psi_{+}(u) \geq \frac{1}{2}\|u\|_{E}^{2}-\frac{\lambda}{q} \int_{\Omega} f(x)\left|u^{+}\right|^{q} d x+\frac{b_{1}}{r} \int_{\Omega} h(x)\left|u^{+}\right|^{r} d x
$$

Taking $k_{1}=\frac{\lambda f(x)}{q}, k_{2}=\frac{b_{1} h(x)}{r}, \eta=q, \beta=r$, and $t=u^{+}$in Lemma 2.3 and integrating over $\Omega$, we obtain

$$
\int_{\Omega}\left(\frac{\lambda f(x)}{q}\left|u^{+}\right|^{q}-\frac{b_{1} h(x)}{r}\left|u^{+}\right|^{r}\right) d x \leq\left(\frac{\lambda}{q}\right)^{\frac{r}{r-q}}\left(\frac{r}{b_{1}}\right)^{\frac{q}{r-q}} \int_{\Omega}\left(\frac{f^{r}}{h^{q}}\right)^{\frac{1}{r-q}} d x .
$$

It follows from (1.3) that

$$
\frac{\lambda}{q}\left\|u^{+}\right\|_{f, q}^{q}-\frac{b_{1}}{r}\left\|u^{+}\right\|_{h, r}^{r} \leq C_{1}
$$

where $C_{1}=C_{1}(\lambda, r, q, f, h)$ is a constant. Hence $\Psi_{+}(u) \geq \frac{1}{2}\|u\|_{E}^{2}-C_{1}$, that is, $\Psi_{+}$is coercive in $E$.

On the other hand, let $\left\{u_{n}\right\} \subset E$ and $u \in E$ satisfy $u_{n} \rightarrow u$ in $E$ as $n \rightarrow \infty$. The compact embeddings $E \hookrightarrow L_{f}^{q}(\Omega), L_{h}^{r}(\Omega)$ and (3.2) yield

$$
\int_{\Omega} f(x)\left|u_{n}^{+}\right|^{q} d x \rightarrow \int_{\Omega} f(x)\left|u^{+}\right|^{q} d x
$$

and

$$
\int_{\Omega} h(x) G\left(x, u_{n}^{+}\right) d x \rightarrow \int_{\Omega} h(x) G\left(x, u^{+}\right) d x
$$


as $n \rightarrow \infty$, which imply that $\Psi_{+}$is a continuous functional from $E$ to $\mathbb{R}$. The convexity of $\Psi_{+}$is trivial. Therefore $\Psi_{+}$is weakly lower semicontinuous in $E$.

By the Weierstrass theorem we deduce that the functional $\Psi_{+}$has a global minimizer in $E$, say $u_{1}$, that is,

$$
\Psi_{+}\left(u_{1}\right)=\inf _{u \in E} \Psi_{+}(u)
$$

Then, $u_{1}$ satisfies the definition of weak solution (3.3). Choosing $u_{1}^{-} \in E$ as a test function, we find

$$
\begin{gathered}
\iint_{\mathbb{R}^{2 N}} \frac{\left(u_{1}(x)-u_{1}(y)\right)\left(u_{1}^{-}(x)-u_{1}^{-}(y)\right)}{|x-y|^{N+2 s}} d x d y+\int_{\Omega} v(x) u_{1}(x) u_{1}^{-}(x) d x \\
-\lambda \int_{\Omega} f(x)\left|u_{1}^{+}\right|^{q-2} u_{1}^{+} u_{1}^{-} d x-\int_{\Omega} h(x) g\left(x, u_{1}^{+}\right) u_{1}^{-} d x=0 .
\end{gathered}
$$

By the elementary inequality

$$
(a-b)\left(a^{-}-b^{-}\right) \leq-\left(a^{-}-b^{-}\right)^{2},
$$

from (3.6) we have that

$$
\iint_{\mathbb{R}^{2 N}} \frac{\left(u_{1}^{-}(x)-u_{1}^{-}(y)\right)^{2}}{|x-y|^{N+2 s}} d x d y+\int_{\Omega} v(x)\left|u_{1}^{-}\right|^{2} d x \leq 0,
$$

which implies that $u_{1}^{-}=0$, that is, $u_{1} \geq 0$ a.e. in $\Omega$. Hence $u_{1}$ is a weak solution of $\left(P_{\lambda}\right)$.

We may apply similar arguments to prove that the functional $\Psi_{-}$has a minimizer in $E$, that is, that there exists $u_{2} \in E$ such that $\Psi_{-}\left(u_{2}\right)=\inf _{u \in E} \Psi_{-}(u)$. Moreover, we can proceed with $\varphi=u_{2}^{+}$similarly as in (3.4). Noting that $(a-b)\left(a^{+}-b^{+}\right) \geq\left(a^{+}-b^{+}\right)^{2}$, we get that $u_{2} \leq 0$ a.e. in $\Omega$ and that $u_{2}$ is also a weak solution of $\left(P_{\lambda}\right)$.

Step 2. We show that if $\lambda$ is large enough, then $u_{1}$ and $u_{2}$ are nontrivial with $\Psi\left(u_{1}\right)<0$ and $\Psi\left(u_{2}\right)<0$.

Define

$$
\lambda_{+}=\inf _{\substack{u \in E \\\left\|u^{+}\right\|_{f, q}=1}}\left\{\frac{q}{2}\|u\|_{E}^{2}+q \int_{\Omega} h(x) G\left(x, u^{+}\right) d x\right\} .
$$

Let $\lambda>\lambda_{+}$. Then there exists a function $u_{0} \in E$ with $\left\|u_{0}^{+}\right\|_{f, q}=1$ such that

$$
\lambda\left\|u_{0}^{+}\right\|_{f, q}^{q}=\lambda>\frac{q}{2}\left\|u_{0}\right\|_{E}^{2}+q \int_{\Omega} h(x) G\left(x, u_{0}^{+}\right) d x \geq \lambda_{+} .
$$

This inequality implies that

$$
\Psi_{+}\left(u_{0}\right)=\frac{1}{2}\left\|u_{0}\right\|_{E}^{2}-\frac{\lambda}{q} \int_{\Omega} f(x)\left|u_{0}^{+}\right|^{q} d x+\int_{\Omega} h(x) G\left(x, u_{0}^{+}\right) d x<0,
$$

and consequently

$$
\Psi\left(u_{1}\right)=\Psi_{+}\left(u_{1}\right)=\inf _{u \in E} \Psi_{+}(u)<0
$$

in other words, $u_{1}$ is nontrivial. 
In the same way, define

$$
\lambda_{-}=\inf _{\substack{u \in E \\\left\|u^{-}\right\|_{f, q}=1}}\left\{\frac{q}{2}\|u\|_{E}^{2}+q \int_{\Omega} h(x) G\left(x,-u^{-}\right) d x\right\} .
$$

If $\lambda>\lambda_{-}$, then it follows that $u_{2}$ is nontrivial with $\Psi\left(u_{2}\right)=\Psi_{-}\left(u_{2}\right)<0$. So far, by the previous steps, we have showed that, for any $\lambda>\lambda_{+}$, problem $\left(P_{\lambda}\right)$ has a nontrivial positive solution $u_{1}$, whereas for any $\lambda>\lambda_{-}$, problem $\left(P_{\lambda}\right)$ has a nontrivial negative solution $u_{2}$.

Step 3. Now, let

$\lambda_{+}^{*}=\inf \left\{\lambda \in \mathbb{R}:\right.$ problem $\left(P_{\lambda}\right)$ has at least one nontrivial positive solution $\}$.

Clearly, $0 \leq \lambda_{+}^{*} \leq \lambda_{+}$.

Lemma 3.2 For any $\lambda>\lambda_{+}^{*},\left(P_{\lambda}\right)$ admits at least one nontrivial positive solution.

Proof Fix $\lambda>\lambda_{+}^{*}$. By the definition of $\lambda_{+}^{*}$, there exists $\mu \in\left(\lambda_{+}^{*}, \lambda\right)$ such that $\left(P_{\mu}\right)$ has a nontrivial solution $u_{\mu} \in E$ with $u_{\mu}>0$ in $\Omega$. Obviously, $u_{\mu}$ is a sub-solution of $\left(P_{\lambda}\right)$.

On the other hand, consider the minimization problem

$$
\inf _{u \in K\left(u_{\mu}\right)} \Psi(u)
$$

where $K\left(u_{\mu}\right)=\left\{u \in E: u \geq u_{\mu}\right\}$. Note that $K\left(u_{\mu}\right)$ is closed and convex and in turn also weakly closed. By Theorem 6.11 of [32], $\Psi(u)$ attains its infimum in $K\left(u_{\mu}\right)$, that is, there exists $\tilde{u} \geq u_{\mu}$ such that $\Psi(\tilde{u})=\inf _{u \in K\left(u_{\mu}\right)} \Psi(u)$, which also solves the variational inequality

$$
\left\{\begin{array}{l}
\tilde{u} \in K\left(u_{\mu}\right) \\
\Psi^{\prime}(\tilde{u})(\zeta-\tilde{u}) \geq 0 \quad \text { for all } \zeta \in K\left(u_{\mu}\right)
\end{array}\right.
$$

Choosing $\zeta=\tilde{u}+\varphi$ with $\varphi \geq 0$ in $E$, we obtain

$$
\langle\tilde{u}, \varphi\rangle_{E} \geq \lambda \int_{\Omega} f(x)|\tilde{u}|^{q-2} \tilde{u} \varphi d x-\int_{\Omega} h(x) g(x, \tilde{u}) \varphi d x
$$

that is, $\tilde{u}$ is a supersolution to problem $\left(P_{\lambda}\right)$ that dominates $u_{\mu}$.

Thus by the maximum principle (see [33], Lemma 5.2) we get the existence of a nontrivial positive solution $u$ of $\left(P_{\lambda}\right)$ with $u_{\mu} \leq u \leq \tilde{u}$.

Let

$$
\lambda_{-}^{*}=\inf \left\{\lambda \in \mathbb{R}: \text { problem }\left(P_{\lambda}\right) \text { has at least one nontrivial negative solution }\right\} .
$$

We can proceed similarly to prove that, for any $\lambda>\lambda_{-}^{*},\left(P_{\lambda}\right)$ admits at least one nontrivial negative solution.

Now, set

$$
\lambda_{*}=\min \left\{\lambda_{+}^{*}, \lambda_{-}^{*}\right\}
$$


and

$$
\lambda^{*}=\max \left\{\lambda_{+}^{*}, \lambda_{-}^{*}\right\}
$$

We can see that $\lambda_{0}$ exists and $\lambda^{*} \geq \lambda_{*} \geq \lambda_{0} \geq 0$, which completes the proof of the last two statements.

\section{Proof of Theorem 1.2}

\subsection{The proof of statement (i)}

First, if $q>2$, under the weaker assumption $\left(\mathrm{H}_{2}^{\prime}\right)$, the number $\lambda_{0}>0$. In fact, if $u \in E$ is a nontrivial solution of problem $\left(P_{\lambda}\right)$, then taking $\varphi=u$ in (2.1), from (1.2) we have that

$$
\begin{aligned}
\|u\|_{E}^{2} & =\lambda \int_{\Omega} f(x)|u|^{q} d x-\int_{\Omega} h(x) g(x, u) u d x \\
& \leq \lambda \int_{\Omega} f(x)|u|^{q} d x-b_{1} \int_{\Omega} h(x)|u|^{r} d x .
\end{aligned}
$$

Letting $k_{1}=\lambda f(x), k_{2}=b_{1} h(x), \eta=q-2, \beta=r-2$, and $t=u$ in Lemma 2.3, we get that

$$
\lambda f(x)|u|^{q-2}-b_{1} h(x)|u|^{r-2} \leq \lambda^{\frac{r-2}{r-q}}\left(\frac{1}{b_{1}}\right)^{\frac{q-2}{r-q}}\left(\frac{f^{r-2}}{h^{q-2}}\right)^{\frac{1}{r-q}} .
$$

Then it follows from (4.1), (4.2), and Lemma 2.1 that

$$
\begin{aligned}
\|u\|_{E}^{2} & \leq \lambda^{\frac{r-2}{r-q}}\left(\frac{1}{b_{1}}\right)^{\frac{q-2}{r-q}} \int_{\Omega}\left(\frac{f^{r-2}}{h^{q-2}}\right)^{\frac{1}{r-q}}|u|^{2} d x \\
& \leq C_{2_{s}^{*}}^{2} \lambda^{\frac{r-2}{r-q}} b_{1}^{\frac{2-q}{r-q}}\left\|\frac{f^{\frac{r-2}{r-q}}}{h^{\frac{q-2}{r-q}}}\right\|_{L^{\frac{N}{2 s}}(\Omega)}\|u\|_{E}^{2} .
\end{aligned}
$$

Thus

$$
\left(1-C_{2_{s}^{*}}^{2} \lambda^{\frac{r-2}{r-q}} b_{1}^{\frac{2-q}{r-q}}\left\|\frac{f^{\frac{r-2}{r-q}}}{h^{\frac{q-2}{r-q}}}\right\|_{L^{\frac{N}{2 s}(\Omega)}}\right)\|u\|_{E}^{2} \leq 0,
$$

which implies that

$$
\lambda \geq\left(C_{2_{s}^{*}}^{2} b_{1}^{\frac{2-q}{r-q}}\left\|\frac{f^{\frac{r-2}{r-q}}}{h^{\frac{q-2}{r-q}}}\right\|_{L^{\frac{N}{2 s}(\Omega)}}\right)^{\frac{q-r}{r-2}}=M_{0}>0 .
$$

Then $\lambda_{0} \geq M_{0}>0$.

In addition, since $\left(\mathrm{H}_{2}\right)$ is replaced by a weaker assumption $\left(\mathrm{H}_{2}^{\prime}\right)$, the proof of Theorem 1.1 becomes more complicated. The treatment of coercivity of the functional $\Psi_{ \pm}$has to be adapted. Indeed, for any $\delta>0$ and $M>0$, we can decompose $\Omega=X \cup Y \cup Z$ with measurable sets $X, Y, Z$ defined as follows:

$$
\left\{\begin{array}{l}
X=\{x \in \Omega: f(x)<M \text { and } h(x)>\delta\} \\
Y=\{x \in \Omega: f(x)<M \text { and } h(x) \leq \delta\} \\
Z=\{x \in \Omega: f(x) \geq M\}
\end{array}\right.
$$


In Lemma 2.3, setting $t=u^{+}, k_{1}=\frac{\lambda f(x)}{q}, k_{2}=\frac{b_{1} h(x)}{r}, \eta=q$, and $\beta=r$ and integrating over $X$, we have

$$
\begin{aligned}
\int_{X}\left(\frac{\lambda f(x)}{q}\left|u^{+}\right|^{q}-\frac{b_{1} h(x)}{r}\left|u^{+}\right|^{r}\right) d x & \leq\left(\frac{\lambda}{q}\right)^{\frac{r}{r-q}}\left(\frac{r}{b_{1}}\right)^{\frac{q}{r-q}} \int_{X}\left(\frac{f(x)^{r}}{h(x)^{q}}\right)^{\frac{1}{r-q}} d x \\
& \leq C_{2},
\end{aligned}
$$

where $C_{2}=C_{2}(M, \delta, \Omega, \lambda)$ is a constant.

Meanwhile, we apply (2.2) with $\eta=q-2$ and $\beta=r-2$ and integrate over $Y \cup Z$ to derive

$$
\begin{aligned}
& \int_{Y \cup Z}\left(\frac{\lambda f(x)}{q}\left|u^{+}\right|^{q}-\frac{b_{1} h(x)}{r}\left|u^{+}\right|^{r}\right) d x \\
& \leq\left(\frac{\lambda}{q}\right)^{\frac{r-2}{r-q}}\left(\frac{r}{b_{1}}\right)^{\frac{q-2}{r-q}} \int_{Y \cup Z}\left(\frac{f^{r-2}}{h^{q-2}}\right)^{\frac{1}{r-q}}\left|u^{+}\right|^{2} d x \\
& \leq C_{2_{s}^{*}}^{2}\left(\frac{\lambda}{q}\right)^{\frac{r-2}{r-q}}\left(\frac{r}{b_{1}}\right)^{\frac{q-2}{r-q}}\left\|\frac{f^{\frac{r-2}{r-q}}}{h^{\frac{q-2}{r-q}}}\right\|_{L^{\frac{N}{2 s}(Y \cup Z)}}\left\|u^{+}\right\|_{E}^{2} .
\end{aligned}
$$

Since $f, h \in L_{\text {loc }}^{1}(\Omega)$, this results in that $|Z| \rightarrow 0$ as $M \rightarrow \infty$ and, for fixed $M,|Y| \rightarrow 0$ as $\delta \rightarrow 0$. Thus, for any $\varepsilon>0$, we can choose $M$ sufficiently large and then $\delta$ sufficiently small such that

$$
C_{2_{s}^{*}}^{2}\left(\frac{\lambda}{q}\right)^{\frac{r-2}{r-q}}\left(\frac{r}{b_{1}}\right)^{\frac{q-2}{r-q}}\left\|\frac{f^{\frac{r-2}{r-q}}}{h^{\frac{q-2}{r-q}}}\right\|_{L^{\frac{N}{2 s}}(Y \cup Z)}<\varepsilon .
$$

Combining (4.3)-(4.5), we have

$$
\int_{\Omega}\left(\frac{\lambda f(x)}{q}\left|u^{+}\right|^{q}-\frac{b_{1} h(x)}{r}\left|u^{+}\right|^{r}\right) d x \leq C_{2}+\varepsilon\left\|u^{+}\right\|_{E}^{2}
$$

Since $u^{+}=\frac{|u|+u}{2}$, we get

$$
\begin{aligned}
& \iint_{\Omega} \frac{\left|u^{+}(x)-u^{+}(y)\right|^{2}}{|x-y|^{N+2 s}} d x d y \\
& \quad=\iint_{\Omega} \frac{|| u(x)|-| u(y)|+u(x)-u(y)|^{2}}{2^{2}|x-y|^{N+2 s}} d x d y \\
& \quad \leq \iint_{\Omega} \frac{|u(x)-u(y)|^{2}}{|x-y|^{N+2 s}} d x d y .
\end{aligned}
$$

Furthermore, since $\int_{\Omega} v(x)\left|u^{+}(x)\right|^{2} d x \leq \int_{\Omega} v(x)|u(x)|^{2} d x$, it follows that

$$
\left\|u^{+}\right\|_{E}^{2} \leq\|u\|_{E}^{2}
$$

Taking $\varepsilon=\frac{1}{4}$ in (4.6) and using (4.7), we obtain

$$
\Psi_{+}(u) \geq \frac{1}{4}\|u\|_{E}^{2}-C_{2} .
$$

Thus $\Psi_{+}(u)$ is coercive in $E$. In the same way, $\Psi_{-}(u)$ is also coercive in $E$. 
So far, all the necessary conditions are satisfied, and we can follow the same lines as in Sect. 3 to get the results of Theorem 1.1.

\subsection{The cases $\lambda=\lambda_{0}, \lambda_{*}, \lambda^{*}$}

For $\lambda=\lambda_{0}$, let $\left\{\lambda_{n}\right\}$ be a decreasing sequence converging to $\lambda_{0}$, and let $\left\{u_{n}\right\}$ be a corresponding sequence of solutions to problems $\left\{P_{\lambda_{n}}\right\}$. By (4.6) we get

$$
\frac{\lambda_{n}}{q} \int_{\Omega} f(x)\left|u_{n}\right|^{q} d x-\frac{b_{1}}{r} \int_{\Omega} h(x)\left|u_{n}\right|^{r} d x \leq C_{2 n}+\varepsilon\left\|u_{n}\right\|_{E}^{2}
$$

where $C_{2 n}=C_{2}\left(M, \delta, \Omega, \lambda_{n}\right)>0$. Then

$$
\begin{aligned}
\left\|u_{n}\right\|_{E}^{2} & \leq \lambda_{n} \int_{\Omega} f(x)\left|u_{n}\right|^{q} d x-b_{1} \int_{\Omega} h(x)\left|u_{n}\right|^{r} d x \\
& \leq \frac{b_{1} q}{r} \int_{\Omega} h(x)\left|u_{n}\right|^{r} d x+q C_{2 n}+q \varepsilon\left\|u_{n}\right\|_{E}^{2}-b_{1} \int_{\Omega} h(x)\left|u_{n}\right|^{r} d x \\
& =\frac{b_{1}(q-r)}{r} \int_{\Omega} h(x)\left|u_{n}\right|^{r} d x+q C_{2 n}+q \varepsilon\left\|u_{n}\right\|_{E}^{2} \\
& \leq q C_{2 n}+q \varepsilon\left\|u_{n}\right\|_{E}^{2} .
\end{aligned}
$$

Setting $\varepsilon=\frac{1}{2 q}$, we obtain

$$
\left\|u_{n}\right\|_{E}^{2} \leq 2 q C_{2 n} .
$$

Since $\left\{\lambda_{n}\right\}$ is bounded, it follows that $C_{2 n}$ is bounded by a constant independent of $n$. Thus by Lemma 2.1 and 2.2, there exist a subsequence of $\left\{u_{n}\right\}$, still denoted by $\left\{u_{n}\right\}$, and $u_{\lambda_{0}} \in E$ such that

$$
\begin{aligned}
& u_{n} \stackrel{\text { weakly }}{\longrightarrow} u_{\lambda_{0}} \quad \text { in } E, \\
& u_{n} \rightarrow u_{\lambda_{0}} \quad \text { in } L_{f}^{q}(\Omega), \\
& u_{n} \rightarrow u_{\lambda_{0}} \quad \text { in } L_{h}^{r}(\Omega) .
\end{aligned}
$$

Since $u_{n}$ are solutions of $\left(P_{\lambda_{n}}\right)$, for all $\varphi \in E$, we have

$$
\left\langle u_{n}, \varphi\right\rangle_{E}-\lambda_{n} \int_{\Omega} f(x)\left|u_{n}\right|^{q-2} u_{n} \varphi d x-\int_{\Omega} h(x) g\left(x, u_{n}\right) \varphi d x=0 .
$$

Passing to the limit as $n \rightarrow \infty$, we deduce that $u_{\lambda_{0}}$ solves problem $\left(P_{\lambda_{0}}\right)$.

We finally claim that $u_{\lambda_{0}} \not \equiv 0$. Indeed, taking $\varphi=u_{\lambda_{0}}$ in the definition of weak solution for $u_{\lambda_{0}}$, we have from (1.2) and Lemma 2.2 that

$$
\begin{aligned}
\left\|u_{\lambda_{0}}\right\|_{E}^{2} & \leq \lambda_{0}\left\|u_{\lambda_{0}}\right\|_{f}^{q}-b_{1}\left\|u_{\lambda_{0}}\right\|_{h}^{r} \\
& \leq \lambda_{0}\left\|u_{\lambda_{0}}\right\|_{f}^{q} \leq \lambda_{0} C_{f}\left\|u_{\lambda_{0}}\right\|_{E}^{q} .
\end{aligned}
$$

Taking into account that $q>2$, we obtain

$$
\left\|u_{\lambda_{0}}\right\|_{E} \geq\left(\frac{1}{\lambda_{0} C_{f}}\right)^{\frac{1}{q-2}}>0,
$$

which implies $u_{\lambda_{0}} \not \equiv 0$. Hence problem $\left(P_{\lambda_{0}}\right)$ admits at least one nontrivial solution. 
For $\lambda=\lambda_{*}$, without loss of generality, we can assume that $\lambda_{*}=\lambda_{+}^{*}<\lambda_{-}^{*}$. Then there also exists a decreasing sequence $\left\{\lambda_{n}\right\}$ converging to $\lambda_{*}$. Let $u_{n} \in E$ be a nontrivial positive solution of problem $\left(P_{\lambda_{n}}\right)$. It suffices to repeat the previous arguments to conclude that $\left(P_{\lambda_{*}}\right)$ has at least one nontrivial positive solution.

For $\lambda=\lambda^{*}$, assuming that $\lambda^{*}=\lambda_{+}^{*}>\lambda_{-}^{*},\left\{P_{\lambda_{*}}\right\}$ admits at least one nontrivial positive solution. Furthermore, since $\lambda^{*}>\lambda_{-}^{*}$, we deduce that $\left\{P_{\lambda_{*}}\right\}$ also admits a nontrivial negative solution. So, the second statement of Theorem 1.2 follows.

\subsection{The case $\lambda \geq \tilde{\lambda}$}

Let

$$
\tilde{\lambda}=\max \left\{\lambda_{+}, \lambda_{-}\right\}
$$

For $\lambda>\tilde{\lambda}$, we already know that $\Psi$ has two nontrivial critical points $u_{1} \geq 0$ and $u_{2} \leq 0$. Now let us show that if $q>2$, then $\left(P_{\lambda}\right)$ has two additional nontrivial solutions.

Lemma 4.1 There exist $\rho \in\left(0,\left\|u_{1}\right\|_{E}\right)$ and $\alpha>0$ such that $\Psi_{+}(u) \geq \alpha$ for all $u \in E$ with $\|u\|_{E}=\rho$.

Proof Since $h(x) G(x, \cdot) \geq 0$ in $\Omega$, we have

$$
\begin{aligned}
\Psi_{+}(u) & =\frac{1}{2}\|u\|_{E}^{2}-\frac{\lambda}{q} \int_{\Omega} f(x)\left|u^{+}\right|^{q} d x+\int_{\Omega} h(x) G\left(x, u^{+}\right) d x \\
& \geq \frac{1}{2}\|u\|_{E}^{2}-\frac{\lambda}{q}\left\|u^{+}\right\|_{f, q}^{q} \\
& \geq \frac{1}{2}\|u\|_{E}^{2}-\frac{\lambda}{q}\|u\|_{f, q}^{q} .
\end{aligned}
$$

From Lemma 2.2 we see that

$$
\begin{aligned}
\Psi_{+}(u) & \geq \frac{1}{2}\|u\|_{E}^{2}-\frac{\lambda}{q} C_{f}\|u\|_{E}^{q} \\
& =\|u\|_{E}^{2}\left(\frac{1}{2}-\frac{\lambda}{q} C_{f}\|u\|_{E}^{q-2}\right) \\
& =\rho^{2}\left(\frac{1}{2}-\frac{\lambda}{q} C_{f} \rho^{q-2}\right) .
\end{aligned}
$$

Take $0<\rho<\min \left\{\left\|u_{1}\right\|_{E},\left(\frac{q}{2 C_{f} \lambda}\right)^{\frac{1}{q-2}}\right\}$. Then

$$
\alpha=\rho^{2}\left(\frac{1}{2}-\frac{\lambda}{q} C_{f} \rho^{q-2}\right)>0
$$

satisfies the statement.

Next, we prove that the Palais-Smale condition holds for every sequence $\left\{u_{n}\right\} \subset E$.

Lemma 4.2 Assume that a sequence $\left\{u_{n}\right\} \subset E$ such that $\left\{\Psi_{+}\left(u_{n}\right)\right\}$ is bounded and $\Psi_{+}^{\prime}\left(u_{n}\right) \rightarrow 0$ in $E^{*}$ as $n \rightarrow \infty$. Then $\left\{u_{n}\right\}$ has a convergent subsequence in $E$. 
Proof Since $\Psi_{+}$is coercive, we have that $\left\{u_{n}\right\}$ is bounded in $E$. It is possible to extract a sequence, still relabeled by $\left\{u_{n}\right\}$, such that $\left\{u_{n}\right\}$ converges weakly to some $u$ in $E$. By Lemma 2.2 we obtain

$$
u_{n} \rightarrow u \quad \text { in } L_{f}^{q}(\Omega) \quad \text { and } \quad u_{n} \rightarrow u \quad \text { in } L_{h}^{r}(\Omega) .
$$

Then $\left\{u_{n}\right\}$ is bounded in $L_{f}^{q}(\Omega)$ and $L_{h}^{r}(\Omega)$ with $\left\|u_{n}\right\|_{f, q} \leq C_{3}$ and $\left\|u_{n}\right\|_{h, r} \leq C_{4}$. Observe that

$$
\begin{aligned}
\Psi_{+}^{\prime}\left(u_{n}\right)\left(u_{n}-u\right) & =\left\langle u_{n}, u_{n}-u\right\rangle_{E}-\int_{\Omega} j\left(x, u_{n}^{+}\right)\left(u_{n}-u\right) d x \\
& =\left\langle u_{n}, u_{n}\right\rangle_{E}-\left\langle u_{n}, u\right\rangle_{E}-\int_{\Omega} j\left(x, u_{n}^{+}\right)\left(u_{n}-u\right) d x,
\end{aligned}
$$

where $j\left(x, u_{n}^{+}\right)=\lambda f(x)\left|u_{n}^{+}\right|^{q-2} u_{n}^{+}-h(x) g\left(x, u_{n}^{+}\right)$. First, note that

$$
\left\langle u_{n}, u\right\rangle_{E} \rightarrow\langle u, u\rangle_{E}
$$

In addition, using Hölder's inequality and claim (4.8), we have

$$
\begin{aligned}
\left|\int_{\Omega} j\left(x, u_{n}^{+}\right)\left(u_{n}-u\right) d x\right| & \leq \lambda C_{3}^{q-1}\left\|u_{n}-u\right\|_{f, q}+b_{2} C_{4}^{r-1}\left\|u_{n}-u\right\|_{h, r} \\
& \rightarrow 0 \quad \text { as } n \rightarrow \infty .
\end{aligned}
$$

Since $\Psi_{+}^{\prime}\left(u_{n}\right)\left(u_{n}-u\right) \rightarrow 0$ as $n \rightarrow \infty$, passing to the limit in (4.9), we obtain $\left\|u_{n}\right\|_{E} \rightarrow\|u\|_{E}$ as $n \rightarrow \infty$. Therefore

$$
\begin{aligned}
& \left\|u_{n}-u\right\|_{E}^{2} \\
& =\left\|u_{n}\right\|_{E}^{2}+\|u\|_{E}^{2}-2 \iint_{\mathbb{R}^{2 N}} \frac{\left(u_{n}(x)-u_{n}(y)\right)(u(x)-u(y))}{|x-y|^{N+2 s}} d x d y \\
& \quad-2 \int_{\Omega} v(x) u_{n}(x) u(x) d x \\
& \quad \rightarrow 2\|u\|_{E}^{2}-2\|u\|_{E}^{2}=0
\end{aligned}
$$

as $n \rightarrow \infty$. Then the Palais-Smale condition holds.

As a consequence, the mountain pass theorem guarantees the existence of a critical point $u_{3}$ for $\Psi_{+}$with $\Psi_{+}\left(u_{3}\right) \geq \alpha>0$, which implies that $u_{3}$ is nontrivial and different from $u_{1}$ and $u_{2}$. Moreover, working as we did for $u_{1}$, we can show that $u_{3} \geq 0$. So, $u_{3}$ is a critical point of $\Psi$. The third nontrivial solution of problem $\left(P_{\lambda}\right)$ is obtained.

Similarly, we apply the mountain pass theorem to $\Psi_{-}$to prove the existence of the fourth nontrivial weak solution of problem $\left(P_{\lambda}\right)$ having negative sign and different from the previous ones. Thus, when $\lambda>\tilde{\lambda}$, four nontrivial solutions have been obtained.

If $\lambda=\tilde{\lambda}$, then we can assume that $\tilde{\lambda}=\lambda_{+}>\lambda_{-}$. Let $\lambda_{n}=\tilde{\lambda}+\frac{1}{n}$. Then $\lambda_{n} \rightarrow \tilde{\lambda}$ as $n \rightarrow \infty$ and $\lambda_{n}>\tilde{\lambda}$ for $n \in \mathbb{N}_{+}$. So, for every $n \in \mathbb{N}_{+}$, there exist two nontrivial positive solutions $u_{n_{1}}$ and $u_{n_{2}}$ of problem $\left(P_{\lambda_{n}}\right)$ with $\Psi\left(u_{n_{1}}\right)<0$ and $\Psi\left(u_{n_{2}}\right) \geq \alpha>0$. Proceeding as in Sect. 4.2, 
we get that there exist $u_{\tilde{\lambda}_{1}} \geq 0$ and $u_{\tilde{\lambda}_{2}} \geq 0$ such that $u_{n_{1}} \rightarrow u_{\tilde{\lambda}_{1}}$ in $E$ and $u_{n_{2}} \rightarrow u_{\tilde{\lambda}_{2}}$ in $E$. Furthermore, we can verify that $u_{\tilde{\lambda}_{1}}$ and $u_{\tilde{\lambda}_{2}}$ are two weak solutions of problem $\left(P_{\tilde{\lambda}}\right)$ with $\Psi\left(u_{\tilde{\lambda}_{1}}\right)<0$ and $\Psi\left(u_{\tilde{\lambda}_{2}}\right) \geq \alpha>0$, which implies that $u_{\tilde{\lambda}_{1}} \neq u_{\tilde{\lambda}_{2}}$. On the other hand, since $\tilde{\lambda}>\lambda_{-},\left(P_{\tilde{\lambda}}\right)$ admits two different nontrivial negative solutions. Thus, for $\lambda=\tilde{\lambda},\left(P_{\tilde{\lambda}}\right)$ has at least four nontrivial solutions.

\section{Acknowledgements}

The authors would like to thank the referees for their useful suggestions.

Funding

This work is supported by National Natural Science Foundation of China (Grant Nos. 11501333, 11571208).

\section{Abbreviations}

Not applicable.

Availability of data and materials

Data sharing not applicable to this article as no datasets were generated or analyzed during the current study.

\section{Competing interests}

The authors declare that they have no competing interests.

\section{Authors' contributions}

Both authors contributed equally to the writing of this paper. Both authors read and approved the final manuscript.

\section{Publisher's Note}

Springer Nature remains neutral with regard to jurisdictional claims in published maps and institutional affiliations.

Received: 16 July 2018 Accepted: 24 September 2018 Published online: 01 October 2018

\section{References}

1. Xiang, M.Q., Zhang, B.L., Rǎdulescu, V.: Existence of solutions for perturbed fractional p-Laplacian equations. J. Differ. Equ. 260(2), 1392-1413 (2016)

2. Di Nezza, E., Palatucci, G., Valdinoci, E.: Hitchhiker's quide to the fractional Sobolev spaces. Bull. Sci. Math. 136 $521-573(2012)$

3. Applebaum, D.: Lévy Processes and Stochastic Calculus, 2nd edn. Cambridge Studies in Advanced Mathematics, vol. 116. Cambridge University Press, Cambridge (2009)

4. Bertoin, J.: Lévy Processes. Cambridge Tracts in Mathematics, vol. 121. Cambridge University Press, Cambridge (1996)

5. Laskin, N.: Fractional quantum mechanics and Lévy path integrals. Phys. Lett. A 268(4), 298-305 (2000)

6. Metzler, R., Klafter, J.: The restaurant at the end of random walk: recent developments in the description of anomalous transport by fractional dynamics. J. Phys. A 37(3), 161-208 (2004)

7. Ambrosetti, A., Brézis, H., Cerami, G.: Combined effects of concave and convex nonlinearities in some elliptic problems. J. Funct. Anal. 122(2), 519-543 (1994)

8. Charro, F., Colorado, E., Peral, I.: Multiplicity of solutions to uniformly elliptic fully nonlinear equations with concave-convex right-hand side. J. Differ. Equ. 246(11), 4221-4248 (2009)

9. Colorado, E., Peral, I.: Semilinear elliptic problems with mixed Dirichlet-Neumann boundary conditions. J. Funct. Anal. 199(2), 468-507 (2003)

10. Alama, S., Tarantello, G.: Elliptic problems with nonlinearities indefinite in sign. J. Funct. Anal. 141, 159-215 (1996)

11. Rădulescu, V., Repovš, D.: Combined effects in nonlinear problems arising in the study of anisotropic continuous media. Nonlinear Anal. 75(3), 1524-1530 (2012)

12. Autuori, G., Pucci, P.: Existence of entire solutions for a class of quasilinear elliptic problems. NoDEA Nonlinear Differ Equ. Appl. 20(3), 977-1009 (2013)

13. Autuori, G., Pucci, P.: Elliptic problems involving the fractional Laplacian in $\mathbb{R}^{N}$. J. Differ. Equ. 255(8), $2340-2362$ (2013)

14. Carboni, G., Mugnai, D.: On some fractional equations with convex-concave and logistic-type nonlinearities. J. Differ. Equ. 262(3), 2393-2413 (2017)

15. Xiang, M.Q., Zhang, B.L., Rădulescu, V.: Multiplicity of solutions for a class of quasilinear Kirchhoff system involving the fractional $p$-Laplacian. Nonlinearity 29, 3186-3205 (2016)

16. Pan, N., Zhang, B.L., Cao, J.: Degenerate Kirchhoff-type diffusion problems involving the fractional $p$-Laplacian. Nonlinear Anal., Real World Appl. 27, 56-70 (2017)

17. Pucci, P., Xiang, M.Q., Zhang, B.L.: Existence and multiplicity of entire solutions for fractional $p$-Kirchhoff equations. Adv. Nonlinear Anal. 5, 27-55 (2016)

18. Liang, S.H., Repovš, D., Zhang, B.L.: On the fractional Schrödinger-Kirchhoff equations with electromagnetic fields and critical nonlinearity. Comput. Math. Appl. 75(5), 1778-1794 (2018)

19. Xiang, M., Zhang, B., Zhang, X.: A nonhomogeneous fractional $p$-Kirchhoff type problem involving critical exponent in $\mathbb{R}^{N}$. Adv. Nonlinear Stud. 17, 611-640 (2017)

20. Xiang, M.Q., Molica Bisci, G., Tian, G.H., Zhang, B.L.: Infinitely many solutions for the stationary Kirchhoff problems involving the fractional $p$-Laplacian. Nonlinearity 29, 357-374 (2016) 
21. Caponi, M., Pucci, P.: Existence theorems for entire solutions of stationary Kirchhoff fractional $p$-Laplacian equations. Ann. Mat. Pura Appl. 195, 2099-2129 (2016)

22. Zhang, X., Zhang, B.L., Xiang, M.Q.: Ground states for fractional Schrödinger equations involving a critical nonlinearity. Adv. Nonlinear Anal. 5(3), 293-314 (2016)

23. Zhang, X., Zhang, B.L., Repovš, D.: Existence and symmetry of solutions for critical fractional Schrödinger equations with bounded potentials. Nonlinear Anal. 142, 48-68 (2016)

24. Díaz, J.I., Gómez-Castro, D., Vázquez, J.L.: The fractional Schrödinger equation with general nonnegative potentials. The weighted space approach. Nonlinear Anal. (to appear). https://doi.org/10.1016/j.na.2018.05.001

25. Giacomoni, J., Mukherjee, T., Sreenadh, K.: Positive solutions of fractional elliptic equation with critical and singular nonlinearity. Adv. Nonlinear Anal. 6(3), 327-354 (2017)

26. Giacomoni, J., Kumar Mishra, P., Sreenadh, K.: Fractional elliptic equations with critical exponential nonlinearity. Adv. Nonlinear Anal. 5(1), 57-74 (2016)

27. Abatangelo, N.: Very large solutions for the fractional Laplacian: towards a fractional Keller-Osserman condition. Adv. Nonlinear Anal. 6(4), 383-405 (2017)

28. Mosconi, S., Perera, K., Squassina, M., Yang, Y.: The Brézis-Nirenberg problem for the fractional p-Laplacian. Calc. Var. Partial Differ. Equ. 55, 1-25 (2016)

29. Faria, L., Miyagaki, O., Pereira, F., Squassina, M., Zhang, C.: The Brézis-Nirenberg problem for nonlocal systems. Adv. Nonlinear Anal. 5(1), 85-103 (2016)

30. Caffarelli, L., Silvestre, L.: An extension problem related to the fractional Laplacian. Commun. Partial Differ. Equ. 32(8), 1245-1260 (2007)

31. Molica Bisci, G., Rădulescu, V., Servadei, R.: Variational Methods for Nonlocal Fractional Problems. Encyclopedia of Mathematics and Its Applications, vol. 162. Cambridge University Press, Cambridge (2016)

32. Berger, M.: Nonlinearity and Functional Analysis. Academic Press, New York (1977)

33. Brändle, C., Colorado, E., De Pablo, A.: A concave-convex elliptic problem involving the fractional Laplacian. Proc. R. Soc. Edinb., Sect. A 143(1), 39-71 (2013)

\section{Submit your manuscript to a SpringerOpen ${ }^{\circ}$ journal and benefit from:}

- Convenient online submission

- Rigorous peer review

- Open access: articles freely available online

- High visibility within the field

Retaining the copyright to your article

Submit your next manuscript at $\boldsymbol{\nabla}$ springeropen.com 